\title{
Phylogenetic Relationships between Some Members of the Genera Deleya, Halomonas, and Halovibrio
}

\author{
S. J. DOBSON, * T. A. MCMEEKIN, AND P. D. FRANZMANN \\ Department of Agricultural Science and the Cooperative Research Centre for the Antarctic and Southern \\ Ocean Environment, University of Tasmania, Box 252C, Hobart, Tasmania 7001, Australia
}

\begin{abstract}
The genera Halomonas and Deleya, which constitute the family Halomonadaceae, are difficult to differentiate on the basis of phenotypic and chemotaxonomic attributes. DNA-rRNA hybridization studies have indicated that some Halomonas spp. have the same level of relationship to the type species of the genus Deleya as some Deleya spp. A phylogenetic analysis of the 16S rRNA séquences of seven members of the Halomonadaceae indicated that the members of the genera Halomonas and Deleya do not form separate monophyletic subgroups, confirming the lack of any phylogenetic support for retention of these taxa as separate genera. A phylogenetic analysis of the 16S rRNA sequence of Halovibrio variabilis confirmed that this species belongs in the Halomonadaceae. All of the members of the Halomonadaceae examined and Halovibrio variabilis possess a cytosine residue at position 486 (Escherichia coli numbering), which is an extremely rare attribute among the prokaryotes and has been reported in only one other species, Listonella anguillarum. Several other signature characteristics which define this group in the gamma subclass of the Proteobacteria are identified. The Jukes-Cantor distances between members of the family Halomonadaceae, including Halovibrio variabilis, range from 0.086 to 0.000 (the levels of similarity between the 16S rRNA sequences range from 92.6 to $100 \%$ ). The members of the genera Halomonas, Deleya, and Halovibrio form a monophyletic group and share common chemotaxonomic and phenotypic characteristics. Subgroups containing members of the genera Halomonas, Deleya, and Halovibrio cannot be resolved on the basis of phylogenetic, chemotaxonomic, or phenotypic data. Our data indicate that the members of the genera Halomonas, Deleya, and Halovibrio should be united in a single genus.
\end{abstract}

The genus Halomonas was created to classify a group of moderately halophilic, gram-negative, rod-shaped strains isolated from a saltern. These strains had a $61 \mathrm{~mol} \% \mathrm{G}+\mathrm{C}$ in their DNA and could tolerate salt concentrations between 20 and $32 \%$ (27). The type species was Halomonas elongata. Subsequently, four additional species of the genus Halomonas were described to accommodate groups of strains isolated from estuarine, Antarctic lake, and Dead Sea waters $(11,13,15,16)$. The members of the genus Halomonas had $\mathrm{G}+\mathrm{C}$ values in the range from 59 to $64 \mathrm{~mol} \%$ and were moderately halophilic and/or halotolerant.

The genus Deleya was created to accommodate a group of marine species (4). This genus was created on the basis of a distinct lineage observed in DNA-rRNA hybridization studies and on the basis of chemotaxonomic features. The members of this genus were strictly aerobic and motile by means of flagella, accumulated poly- $\beta$-hydroxybutyrate, had a requirement for $\mathrm{Na}^{+}$, and had DNA G+C contents in the range from 52 to $68 \mathrm{~mol} \%$. The type species was "Deleya aesta". Deleya aquamarina was later shown to be synonymous with "D. aesta" and replaced it as the type species (1). Two additional, moderately halophilic species have been described for strains isolated from saline soils and water bodies $(22,26)$.

A comparison of $16 \mathrm{~S}$ rRNA oligonucleotide catalogs grouped Halomonas elongata and "Flavobacterium halmephilum" as a distinct lineage within the gamma subclass of the purple bacterium phylum, now known as the class Proteobacteria $(24,31)$. " $F$. halmephilum" was transferred to the genus Halomonas as Halomonas halmophila (13). A comparison of the catalogs of Halomonas elongata and

\footnotetext{
* Corresponding author.
}

Halomonas halmophila with the catalogs of Halomonas subglaciescola and "D. aesta" resulted in the description of the family Halomonadaceae to accommodate the genera Halomonas and Deleya (13).

The genera Halomonas and Deleya are difficult to differentiate on the basis of the phenotypic attributes, fatty acid profiles, polar lipid profiles, and respiratory lipoquinones of their members $(5,12,16,23)$. rRNA-DNA hybridization studies have demonstrated that some Halomonas and Deleya species have similar melting temperatures $\left[T_{m(e)}\right.$, the midpoint of thermal melting profiles for strand separation of DNA-DNA or DNA-RNA duplexes] in relation to D. aquamarina (18).

In this study the $16 \mathrm{~S}$ rRNA sequences of six species representing the genera Halomonas and Deleya were determined to clarify the phylogenetic relationships of these organisms and to determine whether generic distinctions within this group of organisms are warranted.

The genus Halovibrio was created to accommodate an obligately halophilic organism with a vibroid morphology isolated from Great Salt Lake (9). The 16S rRNA sequence of Halovibrio variabilis was determined as this organism has been observed to have chemotaxonomic features similar to those of members of the genera Deleya and Halomonas (12).

\section{MATERIALS AND METHODS}

Microorganisms. Halomonas subglaciescola ACAM $12^{\mathrm{T}}$ (= DSM $\left.4683^{\mathrm{T}}\right)(\mathrm{T}=$ type strain) and Halomonas meridiana ACAM $246^{\mathrm{T}}$ (= DSM $5425^{\mathrm{T}}$ ) were obtained from the Australian Collection of Antarctic Microorganisms (ACAM), University of Tasmania, Hobart, Tasmania, Australia. Halomonas elongata DSM $2851^{\mathrm{T}}$ (= ATCC $\left.33173^{\mathrm{T}}\right), D$. aquamarina DSM $30161^{\mathrm{T}}\left(=\mathrm{ATCC} 14400^{\mathrm{T}}\right)$, Deleya marina 
DSM $4741^{\mathrm{T}}\left(=\right.$ ATCC $\left.25374^{\mathrm{T}}\right)$, Deleya halophila DSM $4770^{\mathrm{T}}$, and Halovibrio variabilis DSM $3051^{\mathrm{T}}$ were obtained from the Deutsche Sammlung von Mikroorganismen und Zellkulturen GmbH, Braunschweig, Germany. The strains were grown at $25^{\circ} \mathrm{C}$ on marine broth (Difco) supplemented with agar.

DNA isolation, polymerase chain reaction amplification, and sequencing of polymerase chain reaction products. The methods used to extract DNA and directly sequence the isolated $16 \mathrm{~S}$ ribosomal DNA polymerase chain reaction products have been described previously (6). Sequencing primer 361-341 (Escherichia coli numbering) (7) was used instead of primer 129-109.

Phylogenetic analysis. The $16 \mathrm{~S}$ rRNA sequences of $D$. aquamarina, D. halophila, D. marina, Halomonas elongata, Halomonas subglaciescola, Halomonas meridiana, and Halovibrio variabilis were aligned manually, taking into account secondary structure considerations, with the $16 \mathrm{~S}$ rRNA sequence of Halomonas halmophila ATCC $19717^{\mathrm{T}}$ and the 16S rRNA sequences of the following 38 organisms belonging to the gamma subclass of the Proteobacteria (culture collection strain designations that are identified in the data base are indicated): Acinetobacter sp. strain ATCC 33604, Aeromonas hydrophila ATCC $7966^{\mathrm{T}}$, Alteromonas haloplanktis ATCC 14393, Arhodomonas oleiferhydrans ATCC 49307, Cardiobacterium hominis ATCC $15826^{\mathrm{T}}$, Coxiella burnetii str. Q177, Chromatium tepidum ATCC $43061^{\mathrm{T}}$, Chromatium vinosum ATCC $17899^{\mathrm{T}}$, Citrobacter freundii ATCC 29935, Ectothiorhodospira halochloris ATCC $35916^{\mathrm{T}}$, Ectothiorhodospira halophila str. SL 1, Ectothiorhodospira shaposhnikovii, Erwinia carotovora ATCC 5713, Erwinia herbicola, Escherichia coli, "Flavobacterium lutescens" ATCC 27951, Haemophilus influenzae ATCC $33391^{\mathrm{T}}$, Hafnia alvei ATCC $13337^{\mathrm{T}}$, Legionella pneumophila subsp. pneumophila ATCC $33152^{\mathrm{T}}$, Marinobacter hydrocarbonoclasticus ATCC $49840^{\mathrm{T}}$, Marinomonas vaga ATCC $27119^{\mathrm{T}}$, Methylococcus capsulatus str. BATH, Methylomonas methanica str. 81Z, Oceanospirillum linum ATCC $11336^{\mathbf{T}}$, Pasteurella multocida ATCC $43137^{\mathrm{T}}$, Plesiomonas shigelloides ATCC $14029^{\mathrm{T}}$, Proteus vulgaris IFAM 1731, Pseudomonas aeruginosa ATCC 25330, Pseudomonas mendocina ATCC $25411^{\mathrm{T}}$, Ruminobacter amylophilus ATCC 29477 , Serratia marcescens ATCC $13880^{\mathrm{T}}$, str. symbiont P of Acyrthosyphon pisum, str. symbiont S of Acyrthosyphon pisum, Vibrio parahaemolyticus ATCC $17802^{\mathrm{T}}$, Wolbachia persica ATCC VR 331, Xanthomonas maltophilia ATCC $13637^{\mathrm{T}}$, Xylella fastidiosa ATCC 35880 , and Yersinia enterocolitica ATCC $9610^{\mathrm{T}}$. The sequences of most of these species were obtained from the rRNA database project (21); the exceptions were the sequences of Alteromonas haloplanktis, Marinobacter hydrocarbonoclasticus, and Marinomonas vaga, which were obtained from GenBank and then aligned in the rRNA database project format.

Phylogenetic relationships were estimated by distance analysis by using the PHYLIP version 3.4, package. JukesCantor evolutionary distances were calculated by using the DNADIST program, and a phylogenetic tree was derived by using the FITCH program, random order input of sequences, and the global rearrangement option (8). Parsimony analysis was done by using the branch and bound method available in PAUP version 3.0 (25). Both the parsimony and distance trees were unrooted with Pasteurella multocida as the outgroup. Bootstrap analysis (2,000 replications) was performed for both the distance and parsimony methods. The parsimony bootstrap analysis was performed by using a heuristic search method because of computing limitations.
TABLE 1. Positions at which the 16S rRNA sequence of

Halomonas elongata determined in this study differed from the sequence determined by Gauthier et al.

\begin{tabular}{ccc}
\hline $\begin{array}{c}\text { Position(s) } \\
(E . \text { coli } \\
\text { numbering) }\end{array}$ & \multicolumn{2}{c}{ Residue(s) as determined by: } \\
\cline { 2 - 3 } & This study & Gauthier et al. \\
\hline 184 & $\mathrm{C}$ & \\
932 & $\mathrm{C}$ & \\
$1003-1004$ & $\mathrm{GA}$ & $\mathrm{GGA}$ \\
1009 & $\mathrm{~T}$ & $\mathrm{C}$ \\
1168 & $\mathrm{C}$ & $\mathrm{T}$ \\
1208 & $\mathrm{C}$ & $\mathrm{A}$ \\
1403 & $\mathrm{C}$ & AGTCACA \\
$1461-1467$ & ATCACCA &
\end{tabular}

${ }^{a}$ Data from reference 14.

Nucleotide sequence accession numbers. The sequences determined in this study have been deposited in the GenBank data base under accession numbers M93352 through M93358.

\section{RESULTS}

The number of bases determined for the 16S rRNA genes of the seven species ranged from 1,402 to 1,458 (average, 1,432 ), which represented $96.2 \%$ of the sequence in the region from position 29 to position 1521 (Escherichia coli numbering). The sequence of Halomonas elongata ATCC $33173^{\mathrm{T}}$ was compared with the sequence obtained by Gauthier et al. (14). There were nine differences between the two sequences (Table 1). A reexamination of the sequencing autoradiographs resolved the differences in favor of the results of this study.

The 16S rRNA sequences of all of the members of the Halomonadaceae which we examined had a cytosine residue at 486 , which is a highly distinctive signature. An examination of 436 sequences in the rRNA database project (21) did not reveal any organism that possessed a $C$ residue at this site. According to a recent description of the partial 16S rRNA sequence of Listonella anguillarum, this organism also contains a $\mathrm{C}$ at this position (19). The closest relatives of Listonella anguillarum, Vibrio fischeri and Vibrio logei, do not contain a cytosine residue at this position (19). Most other members of the Bacteria possess a uracil residue; the exception is Planctomyces staleyi, which has an adenine residue. A deletion occurs in members of the Archaea.

A comparison of the sequences of the members of the Halomonadaceae with the sequences of 38 other members of the gamma subclass of the Proteobacteria revealed several characteristics which formed a distinctive signature for the Halomonadaceae within the gamma subclass (Table 2). Other members of the gamma subclass contained a maximum of 5 of the 17 characteristics identified. With one exception (Listonella anguillarum), in the gamma subclass the signature at position 486 met the stringent condition of having a constant composition among members of the Halomonadaceae and a different and constant composition in all other members of the subclass (29). Similarly, for the signature at position 776 there was only one other known member of the gamma subclass which had the same composition as the members of the Halomonadaceae; all other members of the subclass had a different and constant composition. 
TABLE 2. Signature characteristics of the 16S rRNA gene for members of the Halomonadaceae and frequency of occurrence among other members of the gamma subclass of the Proteobacteria

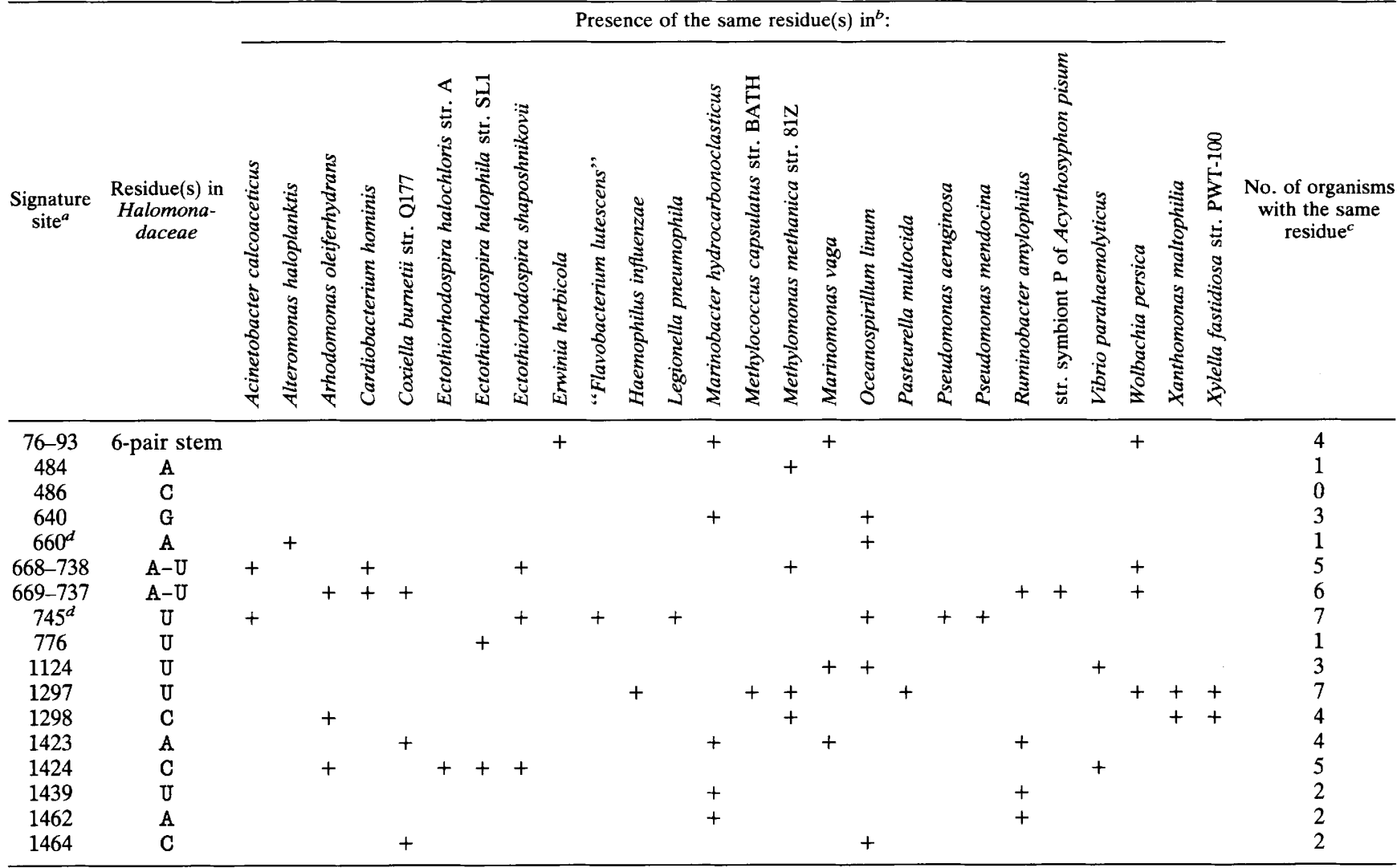

${ }^{a}$ E. coli numbering.

${ }^{b}$ Most sequences were obtained from the rRNA database project (21). The exceptions were the Alteromonas haloplanktis, Marinobacter hydrocarbonoclasticus, and Marinomonas vaga sequences, which were obtained from GenBank.

${ }^{c}$ The number of organisms belonging to the gamma subclass of the Proteobacteria out of a sample of 38 available sequences (excluding members of the Halomonadaceae) which contained the same 16S rRNA signature characteristic as the members of the Halomonadaceae.

${ }^{d}$ Residues 660 and 745 are paired.

Table 3 shows the evolutionary distances calculated by the Jukes-Cantor equation and the levels of sequence similarity between members of the genera Deleya, Halomonas, and Halovibrio and some representatives of the gamma subclass of the Proteobacteria. D. aquamarina and Halomonas meridiana were the most highly related organisms, having identical sequences over the determined region. The distances between other members of the three genera ranged from 0.026 to 0.086 , and the levels of sequence similarity ranged from 92.5 to $97.6 \%$. The closest relative of members of the Halomonadaceae was $O$. linum. The evolutionary distances between $O$. linum and members of the Halomonadaceae ranged from 0.11 to 0.125 , and the levels of sequence similarity ranged from 88.8 to $90.4 \%$.

The phylogenetic tree derived by distance analysis is shown in Fig. 1. Two trees of equal minimal length were found by parsimony analysis (Fig. 2). One of these (Fig. 2b) had a topology similar to that of the tree derived from distance analysis. The topology of the tree in Fig. $2 b$ also was the result obtained from a distance and parsimony analysis of a conserved data set, in which positions at which less than $50 \%$ of the taxa had the same nucleotide were eliminated from the data set. The members of the genera Halomonas and Deleya did not form separate monophyletic subgroups in the phylogenetic trees. This confirmed that there is no phylogenetic basis to distinguish the two genera and was consistent with results obtained from rRNA-DNA (18), chemotaxonomic $(12,23)$, and phenotypic $(5,16)$ studies.

Halovibrio variabilis clustered with the members of the Halomonadaceae (Fig. 1 and 2) and was most closely related to $D$. aquamarina and Halomonas meridiana, with a level of 16S rRNA sequence similarity of $96.7 \%$. Halovibrio variabilis was clearly a member of the Halomonadaceae and is treated as such below.

The members of the Halomonadaceae were separated into the following three clusters on the distance tree (Fig. 1) and one of the trees derived from parsimony analysis (Fig. 2b): (i) cluster 1 , containing $D$. aquamarina, Halomonas meridiana, Halovibrio variabilis, and Halomonas subglaciescola; (ii) cluster 2, containing Halomonas elongata, Halomonas halmophila, and D. halophila; and cluster 3 , containing $D$. marina. However, bootstrap analyses in which both the parsimony and distance methods were used indicated that the internal topology of the Halomonadaceae group was not stable (Fig. 3). Strong support existed only for a $D$. aquamarina-Halomonas meridiana-Halovibrio variabilis subcluster within cluster 1 described above. 
TABLE 3. 16S rRNA evolutionary distances as calculated by the Jukes-Cantor equation and levels of 16S rRNA similarity for members of the genera Deleya, Halomonas, and Halovibrio and some other members of the gamma subclass of the Proteobacteria

\begin{tabular}{|c|c|c|c|c|c|c|c|c|c|c|c|c|c|c|c|}
\hline \multirow[b]{2}{*}{ Organism } & \multicolumn{15}{|c|}{ Evolutionary distance or level of $16 \mathrm{~S}$ rRNA similarity $(\%)^{a}$} \\
\hline & 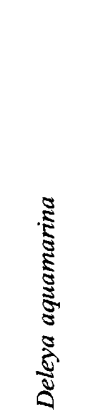 & 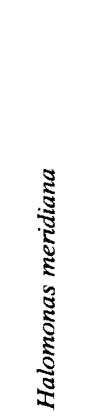 & 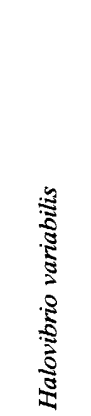 & 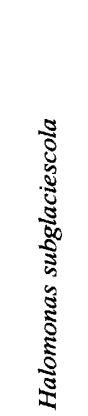 & 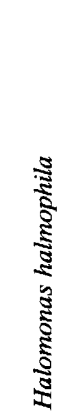 & 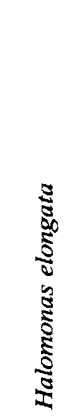 & 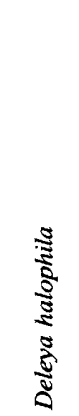 & 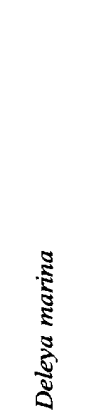 & 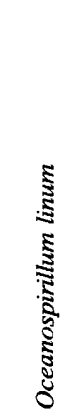 & 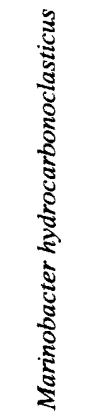 & 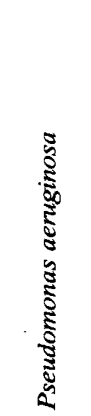 & 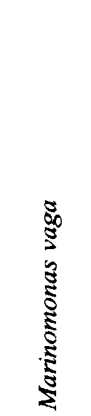 & 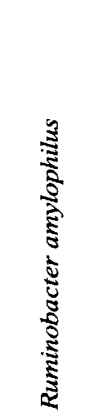 & 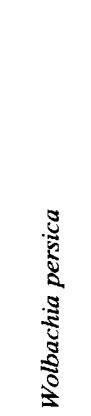 & 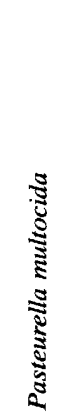 \\
\hline Deleya aquamarina & & 100 & 96.7 & 95.3 & 94.0 & 94.0 & 94.0 & 93.6 & 89.5 & 88.4 & 88.2 & 87.5 & 85.3 & 84.9 & 82.8 \\
\hline Halomonas meridiana & 0.000 & & 96.7 & 95.0 & 93.7 & 93.8 & 93.9 & 93.6 & 89.1 & 88.2 & 88.0 & 87.2 & 85.0 & 84.6 & 82.7 \\
\hline Halovibrio variabilis & 0.036 & 0.035 & & 94.5 & 92.5 & 93.1 & 94.0 & 92.6 & 88.8 & 88.1 & 87.5 & 86.9 & 84.4 & 83.1 & 82.6 \\
\hline Halomonas subglaciescola & 0.051 & 0.053 & 0.059 & & 94.8 & 94.8 & 94.5 & 93.2 & 89.2 & 88.1 & 87.4 & 88.1 & 83.8 & 83.2 & 82.0 \\
\hline Halomonas halmophila & 0.065 & 0.068 & 0.082 & 0.055 & & 97.6 & 95.7 & 93.5 & 89.0 & 86.8 & 87.3 & 87.3 & 83.3 & 83.0 & 81.9 \\
\hline Halomonas elongata & 0.066 & 0.068 & 0.075 & 0.056 & 0.025 & & 96.7 & 94.1 & 89.4 & 87.6 & 87.7 & 87.7 & 83.5 & 83.5 & 82.1 \\
\hline Deleya halophila & 0.068 & 0.069 & 0.068 & 0.061 & 0.047 & 0.036 & & 95.2 & 90.2 & 88.7 & 87.8 & 88.5 & 84.0 & 83.7 & 82.7 \\
\hline Deleya marina & 0.073 & 0.073 & 0.085 & 0.077 & 0.073 & 0.067 & 0.056 & & 90.4 & 89.0 & 87.9 & 88.2 & 84.7 & 84.1 & 82.8 \\
\hline Oceanospirillum linum & 0.118 & 0.121 & 0.125 & 0.120 & 0.120 & 0.118 & 0.111 & 0.110 & & 89.7 & 88.4 & 89.4 & 84.2 & 84.2 & 83.8 \\
\hline Marinobacter hydrocarbonoclasticus & 0.132 & 0.133 & 0.134 & 0.134 & 0.148 & 0.140 & 0.132 & 0.128 & 0.113 & & 88.1 & 88.3 & 84.1 & 83.6 & 82.2 \\
\hline Pseudomonas aeruginosa & 0.133 & 0.134 & 0.141 & 0.142 & 0.140 & 0.137 & 0.142 & 0.140 & 0.127 & 0.133 & & 88.2 & 86.0 & 84.3 & 82.6 \\
\hline Marinomonas vaga & 0.142 & 0.145 & 0.149 & 0.132 & 0.142 & 0.138 & 0.133 & 0.137 & 0.115 & 0.130 & 0.130 & & 84.3 & 85.3 & 83.8 \\
\hline Ruminobacter amylophilus & 0.170 & 0.171 & 0.180 & 0.186 & 0.191 & 0.190 & 0.192 & 0.183 & 0.178 & 0.182 & 0.156 & 0.178 & & 82.8 & 85.3 \\
\hline Wolbachia persica & 0.176 & 0.179 & 0.199 & 0.196 & 0.197 & 0.192 & 0.198 & 0.193 & 0.180 & 0.191 & 0.179 & 0.167 & 0.198 & & 81.7 \\
\hline Pasteurella multocida & 0.203 & 0.202 & 0.204 & 0.211 & 0.210 & 0.210 & 0.211 & 0.210 & 0.185 & 0.208 & 0.201 & 0.185 & 0.164 & 0.214 & \\
\hline
\end{tabular}

${ }^{a}$ The values on the lower left are 16S rRNA evolutionary distances as calculated by the Jukes-Cantor equation, and the values on the upper right are levels of $16 \mathrm{~S}$ rRNA similarity, expressed as percentages.

An examination of the sequence similarity values also suggested that clusters 1 through 3 did not represent unreservedly distinct subgroups within the Halomonadaceae (Table 3). The differences in the distances of Halomonas subglaciescola and $D$. halophila from members of different subgroups were marginal. Halomonas subglaciescola was related to the other members of the $D$. aquamarina subgroup at similarity levels of 94.5 to $95.3 \%$ and to Halomonas elongata and Halomonas halmophila at a similarity level of $94.8 \%$. D. halophila was related to the other members of the Halomonas elongata subgroup at similarity levels of 95.7 to $96.7 \%$ and to $D$. marina at a similarity level of $95.2 \%$. Therefore, Halomonas subglaciescola and D. halophila were not clearly aligned with one subgroup but were intermediate between subgroups.

The inclusion of Halomonas subglaciescola in cluster 1 also was not supported by an examination of the character data. There were more positions at which Halomonas subglaciescola was monophyletic with regard to Halomonas elongata, Halomonas halmophila, and D. halophila than at which it was monophyletic with regard to the other members of cluster 1 . Similarly, the uncertainty about the placement of $D$. halophila was evident from the character data. The number of sequence positions at which the identity of the base in D. halophila was the same as that in $H$. halmophila and $H$. elongata and different from the identities in all other members of the Halomonadaceae was five, compared with the seven positions at which the identity of the base in $D$. halophila was the same as that in $D$. marina and different from the identities in all other members of the Halomonadaceae.

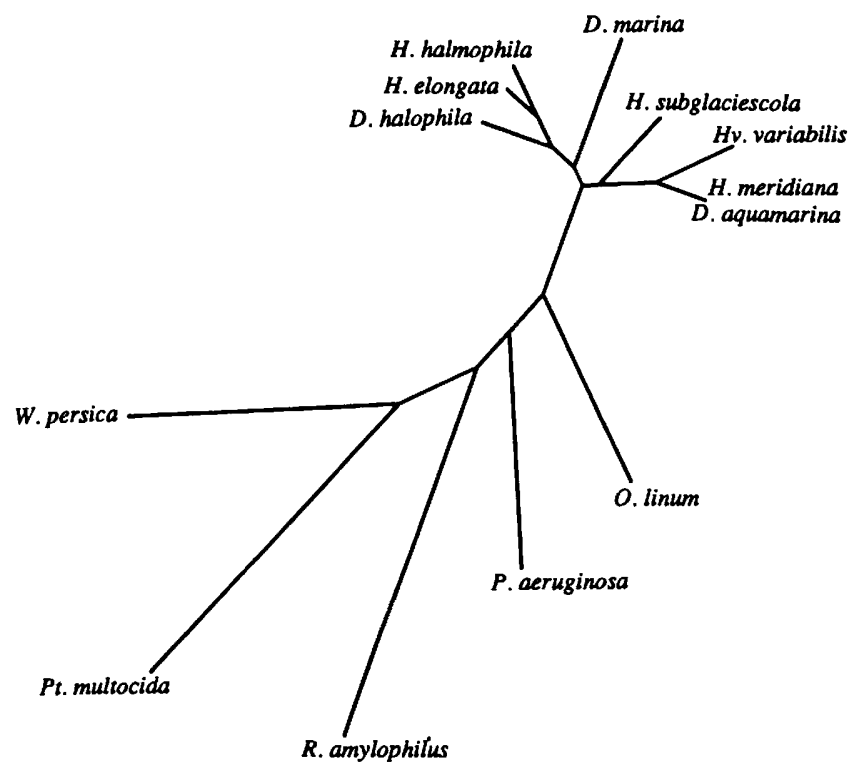

FIG. 1. Phylogenetic tree derived by distance analysis of $16 \mathrm{~S}$ rRNA sequences, showing the relationships among some members of the genera Deleya, Halomonas, and Halovibrio and some other members of the gamma subclass of the Proteobacteria. Pasteurella multocida is the outgroup organism. Bar $=0.03$ evolutionary distance unit as calculated by the Jukes-Cantor equation. 
a

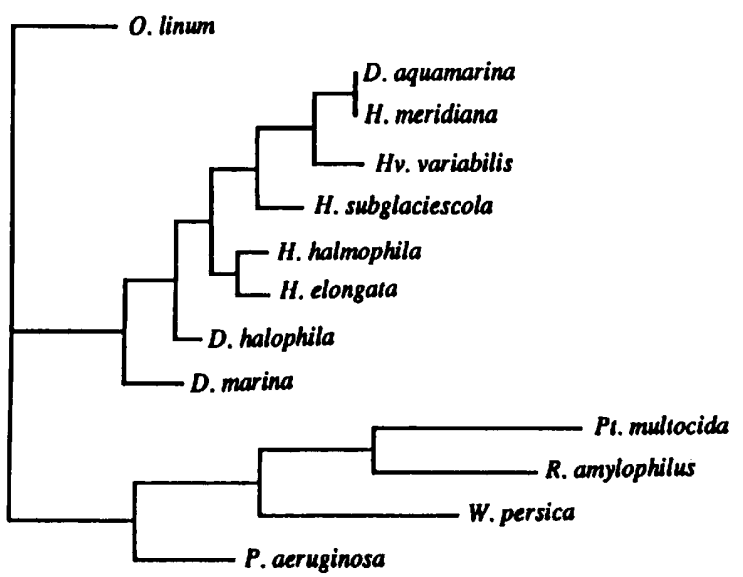

b

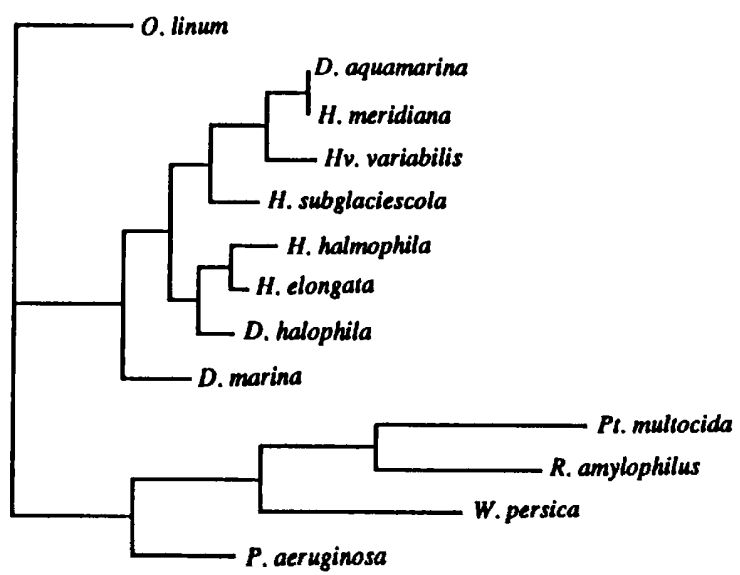

FIG. 2. Equally most parsimonious trees derived from an analysis of the 16S rRNA sequences of some members of the genera Deleya, Halomonas, and Halovibrio and some other members of the gamma subclass of the Proteobacteria. Pasteurella multocida is the outgroup organism. Bar (length of horizontal branches) $=50$ nucleotide differences.

\section{DISCUSSION}

Six oligonucleotides from the catalogs of Halomonas elongata and Halomonas halmophila were identified previously as unique to these organisms within the gamma subclass of the Proteobacteria (31); however, within the wider set of organisms examined in this study, only one of these signatures, occurring at positions 1297 and 1298 (Table 2), had value as a signature for the Halomonadaceae. An additional 16 characteristics present in the 16S rRNA sequences of all members of the genera Deleya, Halomonas, and Halovibrio examined and sparsely distributed among the other members of the gamma subclass of the Proteobacteria provide a defining signature for this family (Table 2). One of these signature characteristics, a cytosine residue at position 486 , was extremely rare among the prokaryotes and has been reported for only one other species, Listonella anguillarum.

The identical 16S rRNA sequences of $D$. aquamarina and Halomonas meridiana suggested that these two names may be synonyms. The $16 \mathrm{~S}$ rRNA data were consistent with the similar fatty acid profiles of the two organisms (Table 4). Halomonas meridiana and " $D$. aesta" (a synonym of $D$. aquamarina) also have identical polar lipid patterns (12). The current, widely accepted definition of a bacterial species is organisms that exhibit levels of DNA-DNA hybridization of approximately $70 \%$ or more and $\Delta T_{m(e)}$ values of $\leq 5^{\circ} \mathrm{C}$ (28).

Amann et al. (2) found that a DNA-DNA hybridization value of $50 \%$ corresponded to a level of $16 \mathrm{~S}$ rRNA sequence similarity of approximately $99 \%$ among strains belonging to the genus Fibrobacter and suggested that genomic sequence conservation varies among groups of strains having similar evolutionary depth as estimated from 16S rRNA sequence similarity data. Fox et al. (10) reported a level of $16 \mathrm{~S}$ rRNA sequence similarity of $99.8 \%$ between two Bacillus sp. which exhibited DNA-DNA hybridization values of 23 to $50 \%$ and concluded that $16 \mathrm{~S}$ rRNA sequence identity within the limits a

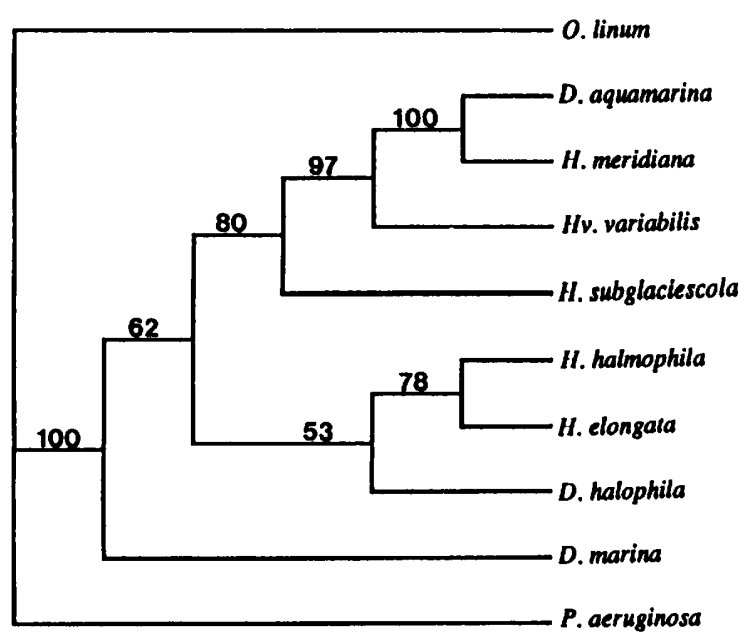

b

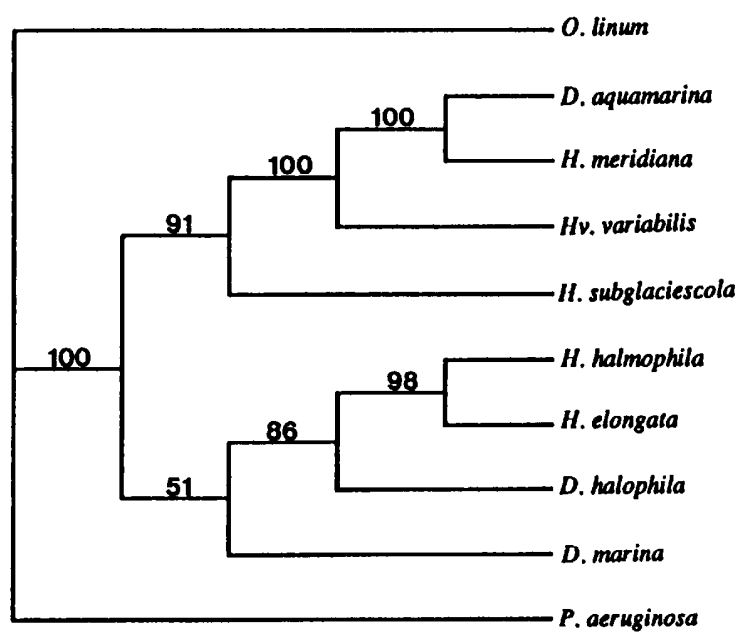

FIG. 3. Bootstrap consensus trees derived from parsimony analysis (a) and distance analysis (2,000 replications) (b), showing the frequency of occurrence of groups where the frequency was more than $50 \%$. 
TABLE 4. Levels of major fatty acids in members of the Halomonadaceae

\begin{tabular}{lccc}
\hline \multicolumn{1}{c}{ Species or strain ${ }^{b}$} & \multicolumn{3}{c}{$\%(\mathrm{wt} / \mathrm{wt})$} \\
\cline { 2 - 4 } & $16: 0$ & $16: 1+$ & $18: 1+$ \\
cyc17:0 & cyc19:0 \\
\hline Halomonas meridiana & 15.5 & 7.7 & 73.4 \\
"Deleya aesta" & 17.0 & 9.1 & 71.2 \\
Deleya aquamarina & 17.4 & 8.4 & 70.7 \\
Deleya venusta & 15.9 & 10.7 & 68.6 \\
Halovibrio variabilis & 17.5 & 13.8 & 66.0 \\
Halomonas halmophila & 20.2 & 11.3 & 64.6 \\
Deleya halophila & 23.3 & 10.6 & 64.6 \\
Halomonas elongata & 26.0 & 9.0 & 62.8 \\
Deleya cupida & 22.6 & 17.5 & 56.1 \\
Deleya pacifica & 26.2 & 15.6 & 55.7 \\
Halomonas subglaciescola & 25.4 & 19.7 & 52.3 \\
Halomonas subglaciescola ACAM 21 & 32.0 & 28.9 & 38.2 \\
Halomonas halodurans & 27.3 & 41.7 & 26.7 \\
Deleya marina & 30.3 & 43.1 & 23.5 \\
\hline
\end{tabular}

${ }^{a}$ Data from reference 12 .

${ }^{b}$ Data for the type strain unless indicated otherwise.

of experimental error is not sufficient to guarantee species identity, where species are defined on the basis of DNADNA hybridization values of $70 \%$ or more. A numerical taxonomic analysis showed that Halomonas meridiana and "D. aesta" were phenotypically distinct (16). Therefore, DNA-DNA hybridization data will be required to clarify whether $D$. aquamarina and Halomonas meridiana are synonyms. Fox et al. (10) state, "In the absence of definitive DNA-DNA data, strains with effectively identical 16S rRNA sequences are best regarded as belonging to the same rRNA species complex or rRNA superspecies. Such strains can be expected to exhibit considerable levels of DNA-DNA homology (typically at least $30 \%$ ), which need not exceed $70 \%$." According to this definition $D$. aquamarina and Halomonas meridiana belong to the same rRNA species complex.

A comparison of DNA-rRNA hybridization values (18) with 16S rRNA similarity values showed that of the species common to both sets of data, Halomonas subglaciescola was the closest to $D$. aquamarina (Table 5). There were

TABLE 5. 16S rRNA similarity values, DNA-rRNA $T_{m(e)}$ values, and DNA-DNA homology values for comparisons between

D. aquamarina DSM $30161^{\mathrm{T}}$ and other members of the genera Halomonas and Deleya

\begin{tabular}{lccc}
\hline \multicolumn{1}{c}{ Organism } & $\begin{array}{c}\text { \% 16S rRNA } \\
\text { similarity }\end{array}$ & $\begin{array}{c}\text { DNA-rRNA } \\
T_{m(e)}\left({ }^{\circ} \mathrm{C}\right)\end{array}$ & $\begin{array}{c}\text { \% DNA-DNA } \\
\text { homology }\end{array}$ \\
\hline Halomonas meridiana & 100 & $-b$ & - \\
Halovibrio variabilis & 96.7 & - & - \\
Deleya venusta & - & 79.0 & 41 \\
Deleya cupida & - & 76.4 & 16 \\
Deleya pacifica & - & 76.6 & 12 \\
Halomonas subglaciescola & 95.3 & 76.2 & - \\
Halomonas halmophila & 94.0 & 76.2 & - \\
Halomonas elongata & 94.0 & 75.6 & - \\
Deleya halophila & 94.0 & 75.2 & - \\
Deleya marina & 93.6 & 75.6 & 12 \\
\hline
\end{tabular}

${ }^{a}$ DNA-rRNA $T_{m(e)}$ values were obtained from reference 18. DNA-DNA homology values were obtained from reference 1 . All values are values for type strains. Values were not available for Halomonas halodurans and Deleya salina.

$b$, data not available. some differences in the arrangement of the other species common to both sets of data with respect to their similarity to D. aquamarina. Halomonas halmophila, Halomonas elongata, and $D$. halophila had the same level of 16S rRNA sequence similarity with respect to $D$. aquamarina, whereas there was a difference of $1^{\circ} \mathrm{C}$ in the $T_{m(e)}$ values of the hybrids. The $T_{m(e)}$ values for Halomonas subglaciescola and Halomonas halmophila were identical, whereas there was a $1.3 \%$ difference in the levels of $16 \mathrm{~S}$ rRNA sequence similarity between these species compared with $D$. aquamarina. In addition, $D$. marina had a higher $T_{m(e)}$ value than $D$. halophila by $0.4^{\circ} \mathrm{C}$, but had a lower level of $16 \mathrm{~S}$ rRNA sequence similarity by $0.4 \%$. These differences were marginal and possibly reflect the use of $23 \mathrm{~S}$ rRNA for the hybridization studies and the small sensitivities of hybridization to the distribution and identity of mismatched bases. The possibility that these differences might have arisen from the presence of undetermined bases in the 16S rRNA sequences was considered. However, the arrangement of $16 \mathrm{~S}$ rRNA sequence similarity values was identical when the values were calculated by using a data set from which all positions where there was an undetermined base in any of the sequences were eliminated (data not shown).

Data for DNA-rRNA hybridization between members of the Halomonadaceae and $D$. aquamarina revealed the presence of two subgroups (18). The species most closely related to $D$. aquamarina $\left[\Delta T_{m(e)}, 3^{\circ} \mathrm{C}\right]$ were its synonyms " $D$. aesta" and Alcaligenes faecalis subsp. homari, Deleya venusta, and the misnamed taxa "Achromobacter viscosum," "Pseudomonas bathycetes," "Agarbacterium alginicum," and "Achrombacter turbidus." Related to D. aquamarina in the second subgroup $\left[\Delta T_{m(e)}, 5\right.$ to $\left.7^{\circ} \mathrm{C}\right]$ were Deleya pacifica, Deleya cupida, Halomonas halmophila, Halomonas subglaciescola, Halomonas elongata, D. marina, $D$. halophila, and the misnamed taxa "Achromobacter halophilus" and "Chromobacterium marismortui." The $16 \mathrm{~S}$ rRNA sequence comparisons done in this study provided information on the relatedness of all combinations of pairs of organisms and not just information on relatedness with respect to a single reference organism. While it appeared from the phylogenetic trees that the members of the Halomonadaceae clustered into three subgroups, bootstrap analysis and an examination of distance and character data showed that these clusters were not well supported. On the basis of this phylogenetic analysis, division of the members of the Halomonadaceae into three genera in accord with the composition of the three clusters could not be justified.

The only group of species in the phylogenetic trees that was well supported by the bootstrap analysis was composed of D. aquamarina, Halomonas meridiana and Halovibrio variabilis, which were related at a $16 \mathrm{~S}$ rRNA sequence similarity level of $96.7 \%$ (Jukes-Cantor values, 0.035 to 0.036 ). If this similarity level were considered appropriate for the formation of a genus, then Halomonas subglaciescola and $D$. marina would be assigned to two separate genera, Halomonas halmophila and Halomonas elongata would be assigned to another separate genus not well supported by the results of the parsimony bootstrap analysis (Fig. 2b), and the position of $D$. halophila would remain ambiguous. $D$. halophila exhibited levels of sequence similarity to the Halomonas halmophila-Halomonas elongata group which ranged from 95.7 to $96.7 \%$ and a level of sequence similarity to $D$. marina of $95.2 \%$, and the clustering of $D$. halophila with either the Halomonas halmophilaHalomonas elongata group or D. marina was not supported by the results of the bootstrap analyses. Notwithstanding the 
uncertainties which would arise from the use of a similarity level of 96 to $96.7 \%$ to define genera within the Halomonadaceae, the D. aquamarina-Halomonas meridiana-Halovibrio variabilis group might be considered a separate genus if its members were phenotypically or chemotaxonomically distinct from the other members of the Halomonadaceae.

In a numerical phenotypic comparison which included Halomonas meridiana, "D. aesta" (a synonym of $D$. aquamarina), Halomonas elongata, Halomonas halmophila, and D. halophila, Halomonas meridiana was more distant from "D. aesta" than it was from the other species of the Halomonadaceae (16). This indicates that for members of the Halomonadaceae relationships derived from numerical phenotypic analyses are not in accord with phylogenetic relationships derived from $16 \mathrm{~S}$ rRNA data. In a study of aerobic marine bacteria, Baumann et al. (3) similarly observed that numerical phenotypic analyses were not necessarily useful for identifying associations above the species level. Therefore, significant phenotypic characteristics can be identified only in light of known phylogenetic relationships. A phenotypic comparison of all members of the Halomonadaceae has not been done; however, an indication of whether phenotypic characteristics might be useful for differentiating the $D$. aquamarina-Halomonas meridianaHalovibrio variabilis subgroup from other members of the Halomonadaceae is given by the phenotypic data of James et al. (16), who studied Halomonas meridiana, "D. aesta," and the type strains of four other members of the Halomonadaceae. These data were examined to identify characteristics which were identical in Halomonas meridiana and " $D$. aesta" and which could differentiate this pair of organisms from the other members of the Halomonadaceae examined. The only attributes identified that might be useful for this purpose were utilization of some sole carbon sources. However, it seems unlikely that carbon source utilization patterns would be useful for defining the $D$. aquamarinaHalomonas meridiana-Halovibrio variabilis group, as one of the characteristic features of Halovibrio variabilis is its inability to grow on a wide range of sole carbon sources (10). For example, in so far as the data of James et al. (16) and Fendrich (9) can be compared, the growth of both " $D$. aesta" and Halomonas meridiana was stimulated by D-glucose, maltose, mannose, and succinate, whereas the growth of Halovibrio variabilis was not.

All members of the Halomonadaceae contained the same major fatty acids (12). Thus, subgroups within the Halomonadaceae could not be characterized by signature fatty acids. The major fatty acids, 16:0, 16:1, 17:0cyc, 18:1, and 19:0cyc, represented 95.2 to $99.1 \%$ of the total fatty acids. The conversion of monounsaturated fatty acids to cyclopropane acids is a one-step synthesis which is sensitive to salt concentration, oxygen tension, growth rate, and temperature (30). The sum of the levels of the monounsaturated fatty acid and its derived cyclopropane fatty acid is a more stable characteristic than the level of the cyclopropane fatty acid alone (17). The relative levels of the fatty acids in the profiles of the members of the Halomonadaceae are shown in Table 4 in decreasing order of the level of 18:1 plus 19:0cyc. In this arrangement the members of the $D$. aquamarina-Halomonas meridiana-Halovibrio variabilis group have higher levels of 18:1 plus 19:0cyc and lower levels of 16:0 than the other members of the Halomonadaceae; however, the differences are marginal. The differences in the levels of 16:0, 16:1 plus 17:0cyc, and 18:1 plus 19:0cyc in Halovibrio variabilis and Halomonas halmophila range from 1 to 3\%. A determination of whether this level of difference is significant would require an estimate of error from repeat determinations of these profiles. If evidence of this difference were repeatable, larger than experimental error, and larger than intraspecies variation, the identification of an unknown strain would still require a concomitant determination of the fatty acid profiles of several reference species of the Halomonadaceae to account for the effect of small differences in culture conditions with each experiment. The creation of a separate genus on the basis of such a small difference in fatty acid profiles is not justified in our opinion. The majority of the minor fatty acid components were present at levels of less than $1 \%$. Utilization of these components to define new genera would similarly be unreliable.

Although the $D$. aquamarina-Halomonas meridianaHalovibrio variabilis cluster was well supported phylogenetically, phenotypic characteristics which defined this group, could not be identified, polar lipid patterns and the identities of respiratory lipoquinones did not discriminate the group from the other members of the Halomonadaceae, and the differences in the proportions of fatty acids were marginal and could not practicably be employed to define the group. The Ad Hoc Committee on Approaches to Taxonomy within the Proteobacteria expressed the opinion that "The first step in the identification of bacteria is the assignment of organisms to genera. Therefore, the greatest clarity in circumscription and utility in the choice of characteristics must be accorded to the level of genera. It is completely impracticable to define genera solely on the basis of phylogenetic data" (20). In accord with the opinion of the Ad Hoc Committee on Approaches to Taxonomy within the Proteobacteria, we concluded that the $D$. aquamarina-Halomonas meridianaHalovibrio variabilis group cannot be described as a separate genus.

Given the possibility that in the future phenotypic or chemotaxonomic characteristics might be identified which could define phylogenetic groups at the genus level among the set of organisms currently assigned to the genera Deleya, Halomonas, and Halovibrio, the identification of well-resolved phylogenetic groups within this set of organisms will remain problematic. The position of $D$. halophila is ambiguous at a similarity level of $96 \%$. At a $16 \mathrm{~S}$ rRNA sequence similarity level of $97 \%$ Halomonas subglaciescola, $D$. halophila, and D. marina would separate into individual groups; Halomonas elongata and Halomonas halmophila would form a cluster (sequence similarity level, 97.6\%) that was not well supported by the results of bootstrap analysis when the parsimony method was used (Fig. 3a); and the D. aquamarina-Halomonas meridiana-Halovibrio variabilis group, which was well supported phylogenetically, would break down, as Halovibrio variabilis, which had a level of $16 \mathrm{~S}$ rRNA sequence similarity to the other two species of $96.7 \%$, would form a separate group.

An examination of the fatty acid profiles of member species of the Halomonadaceae not included in the $16 \mathrm{~S}$ rRNA sequence analysis suggests that the addition of these species to the 16S rRNA phylogenetic tree would result in even less resolution of groups within the tree. The species are arranged in Table 4 in order of decreasing amount of 18:1 plus 19:0cyc. The concomitant trends with respect to the other major fatty acids are increasing amounts of 16:0 and increasing amounts of $16: 1$ plus $17: 0 \mathrm{cyc}$, although these characteristics are not entirely consistent. The levels of 16:1 plus 17:0cyc and 18:1 plus 19:0cyc in ACAM 21 are intermediate in the range of the levels present in $D$. marina and a group of species which includes $D$. halophila, while the level of $16: 0$ is similar to that in D. marina. Given the similarities 
that exist between the arrangement of species in Table 4 and in the phylogenetic trees, Halomonas subglaciescola ACAM 21 may be predicted to be phylogenetically intermediate between $D$. halophila and $D$. marina. The fatty acid profiles of $D$. cupida and $D$. pacifica were most similar to the profile of Halomonas subglaciescola ACAM $12^{\mathrm{T}}$, and the levels of 16:1 plus $17: 0$ cyc and $18: 1$ plus $19: 0$ cyc in these organisms were intermediate in the range for these fatty acids in Halomonas subglaciescola and Halomonas elongata. The exact phylogenetic position of $D$. cupida and $D$. pacifica is not predictable. The profiles of these organisms fall within a group that contains Halomonas halmophila, Halomonas elongata, D. halophila, and Halomonas subglaciescola ACAM $12^{\mathrm{T}}$. The differences in the levels of fatty acid types are not large in this group and show no consistent trends.

The analysis of the 16S rRNA data indicates that the set of organisms assigned to the genera Deleya, Halomonas, and Halovibrio are resolved into coherent phylogenetic groups only at very high levels of $16 \mathrm{~S}$ rRNA sequence similarity $(>97 \%)$. The question then is whether the phenotypic and chemotaxonomic discrimination between organisms within this group at that level is sufficient to warrant their description as several separate genera rather than separate species. The available phenotypic and chemotaxonomic data suggest that separation of this group of organisms into several different genera is not warranted. The species for which $16 \mathrm{~S}$ rRNA sequence data were obtained represent the diversity in this group in terms of phenotypes, habitats, fatty acid profiles, and $\mathrm{G}+\mathrm{C}$ values. An exception may be $D$. venusta, which has the lowest $\mathrm{G}+\mathrm{C}$ value; however, this species is closely related to $D$. aquamarina, as indicated by rRNADNA hybridization data and confirmed by a DNA-DNA hybridization value of $41 \%$ (Table 5).

The members of the genera Deleya, Halomonas, and Halovibrio form a coherent phylogenetic group which is homogeneous with respect to chemotaxonomic features (fatty acids and respiratory lipoquinones), and traditional phenotypic features appear not to be useful for delineating subgroups within this set of organisms. It is our opinion that there is sufficient phylogenetic, chemotaxonomic, and phenotypic data to indicate that the members of these three genera should be united in a single genus. Unification of the genera Deleya, Halomonas, and Halovibrio would stabilize the taxonomy of this group. Until the current unsatisfactory taxonomy is clarified, any newly described species belonging to this group will remain in a state of limbo.

\section{ACKNOWLEDGMENTS}

We thank Harry Burton of the Australian Antarctic Division for his interest in and support for this project.

This study received funding from grants from the Australian Research Council and the Antarctic Science Advisory Committee. S. J. Dobson was supported by an Australian Postgraduate Research Award. P. D. Franzmann was supported by an Australian Research Council Fellowship.

\section{REFERENCES}

1. Akagawa, M., and K. Yamasato. 1989. Synonomy of Alcaligenes aquamarinus, Alcaligenes faecalis subsp. homari, and Deleya aesta: Deleya aquamarinus comb. nov. as the type species of the genus Deleya. Int. J. Syst. Bacteriol. 39:462-466.

2. Amann, R. I., C. Lin, R. Key, L. Montgomery, and D. Stahl. 1992. Diversity among Fibrobacter isolates: towards a phylogenetic classification. Syst. Appl. Microbiol. 15:23-31.

3. Baumann, L., P. Baumann, M. Mandel, and R. D. Allen. 1972. Taxonomy of aerobic marine eubacteria. J. Bacteriol. 110:402429.
4. Baumann, L., R. D. Bowditch, and P. Baumann. 1983. Description of Deleya gen. nov. created to accommodate the marine species Alcaligenes aestus, $A$. pacificus, $A$. cupidus, $A$. venus tus, and Pseudomonas marina. Int. J. Syst. Bacteriol. 33:793802 .

5. Dobson, S. J. 1988. B.Sc. thesis, University of Tasmania, Hobart, Tasmania, Australia.

6. Dobson, S. J., R. R. Colwell, T. A. McMeekin, and P. D. Franzmann. 1993. Direct sequencing of the polymerase chain reaction-amplified 16S rRNA gene of Fiavobacterium gondwanense sp. nov, and Flavobacterium salegens sp. nov., two new species from a hypersaline Antarctic lake. Int. J. Syst. Bacteriol. 43:77-83.

7. Edwards, U., T. Rogall, H. Blöcker, M. Emde, and E. C. Böttger. 1989. Isolation and direct complete nucleotide determination of entire genes. Characterisation of a gene coding for $16 \mathrm{~S}$ ribosomal genes. Nucleic Acids Res. 17:7843-7853.

8. Felsenstein, J. 1989. PHYLIP-phylogeny inference package (version 3.2). Cladistics 5:164-166.

9. Fendrich, C. 1988. Halovibrio variabilis gen. nov. sp. nov., Pseudomonas halophila sp. nov. and a new halophilic coccoid eubacterium from Great Salt Lake, Utah, USA. Syst. Appl. Microbiol. 11:36-43.

10. Fox, G. E., J. D. Wisotzkey, and P. Jurtshuk, Jr. 1992. How close is close: $16 \mathrm{~S}$ rRNA sequence identity may not be sufficient to guarantee species identity. Int. J. Syst. Bacteriol. 42:166-170.

11. Franzmann, P. D., H. R. Burton, and T. A. McMeekin. 1987. Halomonas subglaciescola, a new species of halotolerant bacteria isolated from Antarctica. Int. J. Syst. Bacteriol. 37:27-34.

12. Franzmann, P. D., and B. J. Tindall. 1990. A chemotaxonomic study of members of the family Halomonadaceae. Syst. Appl. Microbiol. 13:142-147.

13. Franzmann, P. D., U. Wehmeyer, and E. Stackebrandt. 1988. Halomonadaceae fam. nov., a new family of the class Proteobacteria to accommodate the genera Halomonas and Deleya. Syst. Appl. Microbiol. 11:16-19.

14. Gauthier, M. J., B. Lafay, R. Christen, L. Fernandez, M. Acquaviva, P. Bonin, and J.-C. Bertrand. 1992. Marinobacter hydrocarbonoclasticus gen. nov., sp. nov., a new extremely halotolerant, hydrocarbon-degrading marine bacterium. Int. J. Syst. Bacteriol. 42:568-576.

15. Hebert, A. M., and R. H. Vreeland. 1987. Phenotypic comparison of halotolerant bacteria: Halomonas halodurans sp. nov., nom. rev., comb. nov. Int. J. Syst. Bacteriol. 37:347-350.

16. James, S. R., S. J. Dobson, P. D. Franzmann, and T. A. McMeekin. 1990. Halomonas meridiana, a new species of extremely halotolerant bacteria isolated from Antarctic saline lakes. Syst. Appl. Microbiol. 13:270-278.

17. Jantzen, E., and K. Bryn. 1985. Whole-cell and lipopolysaccharide fatty acids and sugars of Gram-negative bacteria, p. 145 172. In M. Goodfellow and D. E. Minnikin (ed.), Chemical methods in bacterial systematics. Academic Press, Inc., London.

18. Kersters, K. 1992. The genus Deleya, p. 3189-3197. In A. Balows, H. G. Trüper, M. Dworkin, W. Harder, and K.-H. Schleifer (ed.), The prokaryotes. A handbook on the biology of bacteria: ecophysiology, isolation, identification, applications, vol. 4, 2nd ed. Springer-Verlag, New York.

19. Kita-Tsukamoto, K., H. Oyaizu, K. Nanba, and U. Simudu. 1993. Phylogenetic relationships of marine bacteria, mainly members of the family Vibrionaceae, determined on the basis of 16S rRNA sequences. Int. J. Syst. Bacteriol. 43:8-19.

20. Murray, R. G. E., D. J. Brenner, R. R. Colwell, P. De Vos, M. Goodfellow, P. A. D. Grimont, N. Pfennig, E. Stackebrandt, and G. A. Zavarin. 1990. Report of the Ad Hoc Committee on Approaches to Taxonomy Within the Proteobacteria. Int. J. Syst. Bacteriol. 40:213-215.

21. Olsen, G. J., N. Larsen, and C. R. Woese. 1991. The ribosomal RNA database project. Nucleic Acids Res. 19:2017-2021.

22. Quesada, E., A. Ventosa, F. Ruiz-Berraquero, and A. RamosCormenzana. 1984. Deleya halophila, a new species of moderately halophilic bacteria. Int. J. Syst. Bacteriol. 34:287-292.

23. Skerratt, J. H., P. D. Nichols, C. A. Mancuso, S. R. James, S. J. 
Dobson, T. A. McMeekin, and H. Burton. 1991. The phospholipid ester-linked fatty acid composition of members of the family Halomonadaceae and genus Flavobacterium. A chemotaxonomic guide. Syst. Appl. Microbiol. 14:8-13.

24. Stackebrandt, E., R. G. E. Murray, and H. G. Trüper. 1988 Proteobacteria classis nov., a name for the phylogenetic taxon that includes the "purple bacteria and their relatives." Int. J. Syst. Bacteriol. 38:321-325.

25. Swofford, D. L. 1990. PAUP: phylogenetic analysis using parsimony, version 3.0. Illinois Natural History Survey, Champaign.

26. Valderrama, M. J., E. Quesada, V. Bejar, A. Ventosa, M. C. Guitierrez, F. Ruiz-Berraquero, and A. Ramos-Cormenzana. 1991. Deleya salina sp. nov., a moderately halophilic gramnegative bacterium. Int. J. Syst. Bacteriol. 41:377-384.

27. Vreeland, R. H., C. D. Litchfield, E. L. Martin, and E. Elliot. 1980. Halomonas elongata, a new genus and species of extremely salt-tolerant bacteria. Int. J. Syst. Bacteriol. 30:485495
28. Wayne, L. G., D. J. Brenner, R. R. Colwell, P. A. D. Grimont, O. Kandler, M. I. Krichesky, L. H. Moore, W. E. C. Moore, R. G. E. Murray, E. Stackebrandt, M. P. Starr, and H. G. Trüper. 1987. Report of the Ad Hoc Committee on Reconciliation of Approaches to Bacterial Systematics. Int. J. Syst. Bacteriol. 37:463-464.

29. Weisburg, W. G., Y. Oyaizu, H. Oyaizu, and C. R. Woese. 1985. Natural relationship between bacteroides and flavobacteria. J. Bacteriol. 164:230-236.

30. Wilkinson, S. G. 1988. Fatty acid composition in members of the Pseudomonadaceae, p. 334-337. In C. Ratledge, and S. G. Wilkinson (ed.), Microbial lipids, vol. 1. Academic Press, Inc., London.

31. Woese, C. R., W. G. Weisburg, C. M. Hahn, B. J. Paster, L. B. Zablen, B. J. Lewis, T. J. Macke, W. Ludwig, and E. Stackebrandt. 1985 . The phylogeny of purple bacteria: the gamma subdivision. Syst. Appl. Microbiol. 6:25-33. 